\title{
A STRAIN ENERGY METHOD FOR ELASTIC-PLASTIC ANALYSIS OF NOTCHES UNDER SHEAR LOADING
}

\author{
Zahra Salemi Najafabadi and Daniel Kujawski* \\ Department of Mechanical and Aerospace Engineering \\ Western Michigan University \\ Kalamazoo, MI 49008, USA
}

\begin{abstract}
Neuber's rule and an equivalent strain energy density (ESED) method are two well-known approximate approaches for elastic-plastic notch analysis. Neuber's rule often overestimates whereas the ESED method underestimates the values of notch root stresses and strains. In the present study an approximate method has been proposed, which is based on the strain energy density analysis for notches under shear loading. The proposed method has been assessed against the FEA results from the literature and a good agreement has been observed. Besides predicting notch strains for loading with predominant plastic zone, a significant improvement is found in contrast with those estimated by the Neuber's rule or the ESED method.
\end{abstract}

Key wards: Strain energy density, elastic-plastic analysis, shear loading.

\section{Introduction}

Fracture of components usually initiates at the stress concentrations where local stresses and strains are higher than nominal ones. Therefore, it is important to estimate accurately the local elastic-plastic stresses and strains at the notch-root. Elastic-plastic finite element analysis (FEA) is an accurate method, however it is an expensive and time consuming technique. Hence, approximate methods have been put forward in order to estimate the notch-root strain and stress concentrations. One of the widely used approximate approaches is Neuber's rule proposed in

\footnotetext{
*Corresponding author: daniel.kujawski@wmich.edu
} 
$1961[1]$

$K_{\tau} K_{\gamma}=K_{t}^{2}$

where $K_{t}$ is an elastic stress concentration factor and $K_{\tau}=\tau / \tau_{\text {nom }}$ and $K_{\gamma}=\gamma / \gamma_{\text {nom }}$ are the stress and strain concentration factors, respectively.

It can be noted that Neuber originally derived his rule for out-of-plane shear loading and his rule in the form of Eq. (1) is routinely used also for other loadings such as tension/compression or bending.

Substituting the relations for $K_{\tau}$ and $K_{\gamma}$, into Eq. (1) and after arrangement one gets

$\tau \gamma=K_{t}^{2} \tau_{\text {nom }} \gamma_{\text {nom }}$

or

$\frac{1}{2} \tau \gamma=K_{t}^{2} \frac{\tau_{n o m} \gamma_{n o m}}{2}$

For nominal stresses being smaller than the yield strength, $\tau_{n o m}<\tau_{Y}$, the right hand side (RHS) of Eq. (2b) represents the nominal elastic strain energy density multiplied by $K_{t}^{2}$. A graphical interpretation of the left hand sides (LHSs) of Eqs. (2a) and (2b) are shown in Fig. 1.

It is interesting to note that Neuber proposed a several different relationships (see Reference [2]), from which Eq. (1) is the most widely used. As it is pointed out in [2], a number of expressions derived by Neuber over the years can be unified by the following relationship

$K_{\tau} K_{\gamma}=\psi K_{t}^{2}$

where the unified parameter $\psi$ is equal to:
a) $\Psi=0.5$ for the law published in 1958 ,
b) $\Psi=1$ for the law published in 1961 ,
c) $\Psi=1-0.1 / \mathrm{K}_{\mathrm{t}}+\mathrm{K}_{\tau} / \mathrm{K}_{\mathrm{t}}^{2}-0.9 \mathrm{~K}_{\gamma} / \mathrm{K}_{\mathrm{t}}^{2}$ for the law published in 1985 ,
d) $\Psi=1-1 / K_{t}+K_{\tau} / K_{t}^{2}$ for the law published in 1985 .

The further details and discussions regarding the parameter $\psi$ can be find out in Reference [2]. 
In the recent years, Zappalorto and Lazzarin [3] [4] [5] [6] carried out extensive investigations on the stress and strain fields close to notches utilizing different forms of stress-strain laws. Their results can be also represented by Eq. (3) [2].

Another approach often used for elastic-plastic analysis at notch is the equivalent strain energy density (ESED) method proposed by Molski and Glinka [7] in 1981 originally formulated for tensile stresses and strains. Since this paper considers pure shear loading, Eq. (4) represents the ESED method in terms of the shear stress and strain.

$$
\int \tau d \gamma=K_{t}^{2} \frac{\tau_{n o m} \gamma_{n o m}}{2}
$$

A graphical interpretation of the LHS of Eq. (4) is given in Fig. 2.

Figure 3 illustrates graphically the comparison between the LHSs of Eqs (2b) and (4). An examination of Fig. 3 indicates that in order to satisfy the RHS of Eqs (2b) or (4), which are the same, the LHS of Eq. (2b) would result in larger values of the notch stresses and strains than the LHS of Eq. (4). Moftakhar et al. [8] advocated that Neuber's rule could be considered as the upper limit whereas the ESED method as the lower limit for the actual elastic-plastic behavior of the notch strain.

Recently, Kilambi and Tipton [9] reported elastic-plastic finite element analysis (FEA) simulations for out-of-plane shear loading, analogous to that used by Neuber, considering three different notch geometries and three strain hardening exponents. It is shown that FEA results are between Neuber's and ESED estimations. In addition, Kilambi and Tipton [9] demonstrated that the success of Neuber's rule depends on the material strain hardening exponent. Especially, Neuber's rule performed reasonably well when the strain hardening exponent, $n$, for the Ramberg-Osgood material model is equal 0.2. The Ramberg-Osgood relationship with $n=0.2$ exhibits a similar stress-strain behavior as Neuber's material model used in his 1961 analysis [1]. For lower values of the strain hardening exponent of 0.1 and 0.05 the agreement between FEA results and estimates from Neuber's rule diminished in particular for the lowest value of $n$ equal to 0.05 .

Can be noted that approximate methods for multiaxial stress and strain analysis at notch have been proposed to study fatigue damage under multiaxial loading, e.g. [10] [11]. Recently, Ince et 
al. [12] have developed a computational model to estimate multiaxial elastic-plastic notch stress and strain by using linear elastic FE stresses, for notch components subjected to non-proportional multiaxial loadings. Comparing strain histories results against experimental data shows a reasonable accuracy for six different non-proportional paths. Ince [13] has also compared the predicted local stress and strain from the developed computational model with the elastic plastic FEA results for both monotonic and cyclic non-proportional loadings. The author stated that the experimental or elastic plastic FEA data might be substituted with the proposed model results to predict fatigue life only in the early stage of design analysis. Campagnolo et al. [14] [15] have studied elastic-plastic notch stress and strain distributions under cyclic loading using 3D analysis.

This paper presents a new strain energy approach for the elastic-plastic analysis of notches. The proposed approach is based on the observations published in the literature that the Neuber's rule given by Eq. (1) or (2) usually overestimates whereas the ESED method, Eq. (4), underestimates the notch root elastic-plastic stresses and strains [8].

\section{The Proposed Approach}

Let's assume that a material behavior in shear loading can be represents by the Ramberg-Osgood relationship of the form,

$\gamma=\frac{\tau}{G}+0.002\left(\frac{\tau}{\tau_{Y}}\right)^{1 / n}$

where $G$ is the elastic shear modulus, $\tau_{Y}$ is the yield strength in shear, and $n$ is the strain hardening exponent.

Using decomposition of the total strain into elastic and plastic parts, the LHS of Neuber's rule Eq. (2b), can be written as,

$L H S_{N}=\frac{1}{2} \tau \gamma=\frac{1}{2} \frac{\tau^{2}}{G}+\frac{1}{2} \tau \gamma_{p}$

Similarly, the LHS of the ESED method, Eq. (4), will take the following form 
$L H S_{E S E D}=\int \tau \gamma=\frac{1}{2} \frac{\tau^{2}}{G}+\frac{1}{1+n} \tau \gamma_{p}$

Both Eqs (6) and (7) are illustrated in Figs 4 and 5, respectively.

The difference, $\Delta$, between Eqs (7) and (6) is also shown in Fig. 4, and can be calculated as [16]:

$\Delta=\left(\frac{1}{2} \frac{\tau^{2}}{G}+\frac{1}{1+n} \tau \gamma_{P}\right)-\left(\frac{1}{2} \frac{\tau^{2}}{G}+\frac{1}{2} \tau \gamma_{P}\right)=\frac{1-n}{2(1+n)} \tau \gamma_{P}$

Since the actual notch-root elastic-plastic strains are between estimations from Neuber's rule and the ESED method, Eq. (8) can be generalized as,

$\Delta=\phi \frac{1-n}{2(1+n)} \tau \gamma_{P}$

Using Eq. (9) the proposed left hand side of the modified relation, $(L H S)_{K S}$, analogous to Eqs (6) and (7) is,

$(L H S)_{K S}=\frac{1}{2} \frac{\tau^{2}}{G}+\frac{1}{2} \tau \gamma_{P}+\phi \frac{1-n}{2(1+n)} \tau \gamma_{P}$

By comparison, one can notice the similarity between Eqs (3) and (10).

It can be noted that

$(L H S)_{K S}=(L H S)_{N} \quad$ if $\phi=0$

and

$(L H S)_{K S}=(L H S)_{E S E D} \quad$ if $\phi=1$

with the $\phi$ being bounded between 0 and 1 , which accounts for the dissipation affected by the extent of plastic deformation. In this study, the following two simple relationships for $\phi$ are investigated,

$\phi_{1}=\left(\frac{G_{S}}{G}\right)^{2}$

and 
$\phi_{2}=\frac{G_{S}}{2 G}$

where $G_{S}=\tau / \gamma$ is the secant modulus, which is equal to the elastic shear modulus $G$ if $\tau<\tau_{Y}$. For $\tau>\tau_{Y}$, during plastic deformation, $G_{S}$ would be smaller than $G$. Hence, the ratio of $G_{S}$ over $G$ would be always less or equal one. Figure 6 shows the variation of $\phi_{1}$ and $\phi_{2}$ as a function $G_{S} / G$.

In general, when the applied nominal stress is elastic, i.e. $\gamma_{\text {nom }}=\gamma_{\text {elastic }}=\tau_{\text {nom }} / G$, the RHSs of Neuber's rule, Eq. (2b) and the ESED method, Eq. (4), represent the nominal elastic strain energy density, $\tau_{\text {nom }} \gamma_{\text {nom }} / 2=\tau_{\text {nom }}^{2} / 2 G$ times $\left(K_{t}\right)^{2}$, which can be written as,

$(R H S)_{N \text { or ESED }}=\frac{\left(K_{t} \tau_{n o m}\right)^{2}}{2 G} \quad\left(\tau_{n o m} \leq \tau_{Y}\right)$

Conventional applications of Neuber's rule and ESED method usually assume that nominal material behavior remain elastic. However, when the applied nominal stress, $\tau_{\text {nom }}$, is about or larger than the yield stress, $\tau_{Y}$, nominal plastic strain, $\gamma_{\text {Pnom }}$, occurs and its contribution should be taken into account. Therefore, the RHSs of Neuber's rule and the ESED method given by Eqs (13a) and (13b) respectively have been modified accordingly.

$(R H S)_{N}=\frac{\left(K_{t} \tau_{n o m}\right)^{2}}{2 G}+\frac{1}{2} K_{t}^{2} \tau_{\text {nom }} \gamma_{\text {Pnom }} \quad\left(\tau_{\text {nom }} \geq \tau_{Y}\right)$

$(R H S)_{E S E D}=\frac{\left(K_{t} \tau_{n o m}\right)^{2}}{2 G}+K_{t}^{2} \frac{\tau_{n o m} \gamma_{P n o m}}{1+n} \quad\left(\tau_{n o m} \geq \tau_{Y}\right)$

According to the Ramberg-Osgood relationship, the plastic strain, $\gamma_{P}$, is proportional to $\tau^{1 / n}$. Thus, when the nominal stress is increased by $K_{t}$ the corresponding nominal plastic strain would increase by the factor of $K_{t}^{n}$. As a result, for $\tau_{\text {nom }} \geq \tau_{Y}$ the modified form of Eq. (13a) can be written as,

$(R H S)_{K S}=\frac{\left(K_{t} \tau_{n o m}\right)^{2}}{2 G}+\frac{1}{2} K_{t}^{(n+1)} \tau_{n o m} \gamma_{P n o m}$ 
Hence, the proposed formula for elastic-plastic notches, which accounts for the contribution from both elastic and plastic nominal strain energy densities gets the following form,

$\phi \frac{1-n}{2(1+n)} \tau \gamma_{P}+\frac{1}{2} \tau \gamma_{P}+\frac{1}{2} \frac{\tau^{2}}{G}=\frac{\left(K_{t} \tau_{n o m}\right)^{2}}{2 G}+\frac{1}{2} K_{t}^{(n+1)} \tau_{n o m} \gamma_{P n o m}$

In the above formula $\phi$ can be given by Eqs (11a) or (11b).

\section{Comparison of the Proposed Approach with FEA Results}

For the purpose of validation of the proposed approach, a set of comprehensive FEA results recently published by Kilambi and Tipton [9] for out-of-plane shear loading has been selected. Pertinent material's data, $K_{t}$, and $n$ values reported in Ref. [9] are tabulated in Table 1. Figure 7 illustrates graphically the FE model together with a quadratic hex $(\mathrm{QH})$ element use in analysis.

Table 1: Material properties and notch geometries

\begin{tabular}{|c|c|c|c|c|}
\hline \multirow{2}{*}{$\mathrm{G}=80 \mathrm{GPa}$} & \multirow{2}{*}{$\mathrm{K}_{\mathrm{t}}=1.95,2.4,3.66$} & $\mathrm{n}=0.05$ & $\mathrm{n}=0.1$ & $\mathrm{n}=0.2$ \\
\hline & & $\tau_{Y}=426(\mathrm{MPa})$ & $\tau_{\mathrm{Y}}=414(\mathrm{MPa})$ & $\tau_{Y}=392(\mathrm{MPa})$ \\
\hline
\end{tabular}

Figures $8 \mathrm{a}-8 \mathrm{i}$, the left column of graphs, depict normalized nominal stress versus notch strains calculated by FEA [9] and estimated by four different approximate methods including Neuber's rule, the ESED method, and the proposed approach, Eq. (14), with two different $\phi$ equal to $\left(\frac{G_{S}}{G}\right)^{2}$ and $\left(\frac{G_{S}}{2 G}\right)$. On the other hand, Figs $8 \mathrm{j}-8 \mathrm{r}$, the right column of graphs, depict the percent errors on the estimated notch strains by these approximate methods with respect to FEA results. The FEA results include three different stress concentration factors $K_{t}=1.95,2.4$, and 3.66 and three strain hardening exponents $n=0.05,0.1$ and 0.2 .

As it seen from the graphs at the left and right columns in Fig. 8 that the ESED method underestimates notch strains for all the cases, except for the case of a notch with $\mathrm{K}_{\mathrm{t}}$ equal 1.95 for nominal stress higher than the yield stress. On the other hand, Neuber's rule overestimates notch strains for all the cases studied. 
Predicted notch strains using the proposed formula, Eq. (14), are between those estimated from Neuber's rule and the ESED method. Estimated results by the proposed formula are more accurate than those predicted by Neuber's rule or the ESED method. The careful examination of Fig. 8 indicates that the estimated notch strains by the proposed formula with both $\phi_{1}=\left(\frac{G_{S}}{G}\right)^{2}$ and $\phi_{2}=\frac{G_{S}}{2 G}$ are almost the same.

\section{Discussion}

The graphs at the right column in Fig. 8 indicate that for the nominal stresses lower than $0.8 \tau_{Y}$ the percentage errors on the notch strains by the proposed formula are less than those obtained by Neuber's rule, and they are in the range from $-6 \%$ to $10 \%$. For the nominal stresses higher than $0.8 \tau_{Y}$, the proposed formula starts to underestimate notch strains with reasonable error, which is less than $20 \%$ however, for most cases it is less than $10 \%$. Also predicted notch strains for $\tau>$ $0.8 \tau_{Y}$, by proposed formula are more accurate than those estimated by Neuber's rule. For the notch geometry with $K_{t}=1.95$ and materials with two lowest strain hardening exponents $\mathrm{n}=0.05$ and 0.1 , the notch strains has been reasonably predicted by the proposed Eq. (14) even for nominal stresses higher than $\tau_{Y}$. Overall percentage of error on notch strains predicted by the proposed formula is much lower than that one corresponding to Neuber's rule. Regarding the effect of $\phi$ it seems that for nominal stress lower than $0.8 \tau_{Y}, \phi_{1}$ gives somewhat better estimation than $\phi_{2}$. While for the nominal stresses higher than $0.8 \tau_{Y}$, the predictions with $\phi_{2}$ indicate rather lower error than that with $\phi_{1}$.

In present analysis the net area is used in expressions for the stress and strain concentration factors. When plastic deformation is predominant in the net area, the gross area could be still under elastic deformation, which in turn would partially constraint the nominal elastic-plastic field. Such partial constraint condition can be accounted for by replacing $K_{t}^{1+n}$ in Eq. (14) by $\sqrt{K_{t}^{2} K_{t}^{1+n}}$ which represents the geometric mean for elastic and predominantly plastic nominal 
strains, respectively. Hence, Eqs (13) and (14) with $\phi=\frac{G_{S}}{2 G}$ would take the modified form of Eqs (15) and (16), respectively:

$(R H S)_{K S}=\frac{\left(K_{t} \tau_{n o m}\right)^{2}}{2 G}+\frac{1}{2} \sqrt{K_{t}^{2} K_{t}^{(n+1)}} \tau_{\text {nom }} \gamma_{\text {Pnom }}$

$\phi \frac{1-n}{2(1+n)} \tau \gamma_{P}+\frac{1}{2} \tau \gamma_{P}+\frac{1}{2} \frac{\tau^{2}}{G}=\frac{\left(K_{t} \tau_{n o m}\right)^{2}}{2 G}+\frac{1}{2} \sqrt{K_{t}^{2} K_{t}^{(n+1)}} \tau_{n o m} \gamma_{P n o m}, \quad \phi=\frac{G_{S}}{2 G}$

Figure 8 depicts the percentage error on the predicted notch strain using Neuber's rule, the ESED method and Eq. (16) for three different stress concentration factors and strain hardening exponents. The comparison of Fig. 9 with Fig. 8 for the lowest stress concentration factor $\left(K_{t}=\right.$ 1.95) and three different strain hardening exponents indicates that for nominal stress lower than $0.8 \tau_{Y}$, both Eqs. (16) and (14) predict notch strains with percentage errors less than $\pm 3 \%$. However, for nominal stresses higher than $0.8 \tau_{Y}$, Eq. (16) overestimates with an error less than $8 \%$, on the other hand Eq. (14) underestimates with an error up to $20 \%$. Therefore, Eq. (16) indicates a significant improvement of the notch strain estimation versus Eq. (14) and is conservative for the case of $K_{t}=1.95$. For two other stress concentration factors $\left(K_{t}=2.4\right.$ and 3.66) and three strain hardening exponents, as it can be seen from Figs 8 and 9, both Eqs (16) and (14) have almost the same estimation for the notch strain with a little improvement for nominal stresses about $0.8 \tau_{Y}$ and higher, using Eq. (16). It is seen that either Eq. (14) or Eq. (16) are somewhat non-conservative for nominal stresses higher than $0.8 \tau_{Y}$ for those cases.

\section{Conclusions}

A new energy based method has been developed to predict notch strain under out-of-plane shear loading. The left hand side of the proposed formula is a summation of three different parts of the strain energies including: $\frac{1}{2} \frac{\tau^{2}}{G}, \frac{1}{2} \tau \gamma_{P}$ and $\phi \frac{1-n}{2(1+n)} \tau \gamma_{P}$. Here, two different relationship for $\phi$ have been studied as $\phi_{1}=\left(\frac{G_{S}}{G}\right)^{2}$ and $\phi_{2}=\frac{G_{S}}{2 G}$. 
The right hand side of the proposed formula, Eq. (14), is similar to the RHS of Neuber's rule, Eq. (13a), the only difference is using $K_{t}^{(n+1)} \tau \gamma_{P}$ instead of $K_{t}^{2} \tau \gamma_{P}$.

Results show a fairly good agreement between the estimated notch strains by Eq. (14) and the FEA results. The estimated values given by Eq. (14) are between those predicted by Neuber's rule and the ESED method. Percentage of errors on predicted notch strains by the proposed method are less than those estimated from Neuber's rule. Especially, for the notch with $K_{t}=1.95$ and the nominal stresses higher than the yield resulting in the predominant nominal plastic deformation. In this case the proposed formula underestimates and Neuber's rule overestimates notch strains. The underestimate notch strains are with an error less than $20 \%$ for the material with the lowest strain hardening exponent $(n=0.05)$ and with errors less than $10 \%$ for material strain hardening exponents of 0.1 and 0.2 . It can be noted that predicted results using both $\phi_{1}=\left(\frac{G_{S}}{G}\right)^{2}$ and $\phi_{2}=\frac{G_{S}}{2 G}$ are almost the same. However for $\tau_{n o m}<0.8 \tau_{Y}, \phi_{1}$ results in somewhat better estimation than $\phi_{2}$. Although, for $\tau_{n o m}>0.8 \tau_{Y}$ the results are invers and $\phi_{2}$ gives a better prediction on notch strain.

An additional modification of the proposed Eq. (14) has been studied by considering the mutual interaction between the predominantly plastic deformation of the net area and an apparent elastic deformation in the gross area. This interaction is accounted by using $\frac{1}{2} \sqrt{K_{t}^{2} K_{t}^{(n+1)}} \tau_{\text {nom }} \gamma_{\text {Pnom }}$ instead of $\frac{1}{2} K_{t}^{(n+1)} \tau_{n o m} \gamma_{\text {Pnom }}$ in the RHS of the modified formula given by Eq. (16). A comparison has been done on estimated notch strains given by Eq. (14) and its modified form, Eq. (16). It is shown, that modified formula, Eq. (16), is conservative for the bluntest notch $\left(\mathrm{K}_{\mathrm{t}}=\right.$

1.95) although is non-conservative when $\tau_{\text {nom }}>0.8 \tau_{Y}$. However, for $\tau_{\text {nom }}>0.8 \tau_{Y}$ the modified formula estimates the notch strain more accurate than Eq. (14). For higher stress concentration factors $\left(K_{t}=2.4\right.$ and 3.66) both formulas provide almost the same notch strains and for $\tau_{\text {nom }}>$ $0.8 \tau_{Y}$ the modified formula, Eq. (16), gives slightly more accurate estimations.

\section{Acknowledgments}

This study is supported by the Office of Naval Research, grant N000141010577. 


\section{References}

[1] Neuber H., "Theory of stress concentration for shear strained prismatical bodies with arbitrary nonlinear stress-strain law.," J Appl Mech: T ASME, vol. 28, pp. 544-550, 1961.

[2] Zappalorto M, Kujawski D., "Neuber's rules and other solutions: Theoretical differences, formal analogies and energy interpretations.," Theoretical and Applied Fracture Mechanics., 2015.

[3] Zappalerto M, Lazzarin P., "Analytical study of the elastic-plastic stress fields ahead of parabolic notches under antiplane shear loading.," Int. J. Fract, vol. 148, pp. 139-154, 2007.

[4] Zappaleto M, Lazzarin P., "A new version of the Neuber rule accounting for the influence of the notch opening angle for out-of-plane shear loads," Int. J. Solids Struct., vol. 46, pp. 1901-1910, 2009.

[5] Zappaleto M, Lazzarin P., "A unified approch to the analysis of nonlinear stress and strain fields ahead of mode III-loaded notches and cracks,", Int. J. Solid Struct, vol. 47, pp. 851-864, 2010.

[6] Zappaleto M, Lazzarin P,. "Some remarks on the Neuber rule applied to a control volume surronding sharp and blunt notch tips,," Fatigue Fract, Eng, Mater, Struct,, vol. 37, pp. 349-358, 2014.

[7] Moleski K, Glinka G., "A method of elastic-plastic stress and strain calculation at a notch root.," Material science and engineering., vol. 50, pp. 93-100, 1981.

[8] Moftakhar A, Buczynski A, Glinka G., "Calculation of elasto-plastic strains and stresses in notches under multiaxial loading.," Int. J. Fract, vol. 70, pp. 357-373, 1995.

[9] Kilambi S, Tipton S. M., "Numerical evaluation of the original "Neuber's rule" for pure out of plane shear loading," JSA, vol. 48(8), pp. 522-535, 2013.

[10] Hoffmann M, Seeger T., "A generalized method for estimating multiaxial elastic-plastic notch stresses and strains, Part 1: Theory.," Journal of Engineering Materials and Technology, vol. 107(4), pp. 250-254., 1985.

[11] Hoffmann M, Seeger T., "Stress-strain analysis and life predictions of a notched shaft under multiaxial loading.," Multiaxial Fatigue-Analysis and Experiments, pp. 81-100., 1989.

[12] Ince A, Glinka G and Buczynski A., "Computational modeling of multiaxial elasto-plastic stressstrain response for notched components under non-proportional loading.," International Journal of Fatigue, vol. 62, pp. 42-52., 2014.

[13] Ince A., "Numerical validation of computational stress and strain analysis model for notched components subject to non-proportional loadings.," Theoretical and Applied Fracture Mechanics , 
2015.

[14] Marangon C, Campagnolo A and Berto F., "Three-dimensional effects at the tip of rounded notches subjected to mode-I loading under cyclic plasticity.," The Journal of Strain Analysis for Engineering Design, vol. 50(5), pp. 299-313., 2015.

[15] Campagnolo A, Berto F and Marangon C., "Cyclic plasticity in three-dimensional notched components under in-phase multiaxial loading at $\mathrm{R}=-1$.," Theoretical and Applied Fracture Mechanics, vol. 81, pp. 76-88, 2016.

[16] Ye D, Matsuoka S, Naoyuki S, Yosho M., "Further investigation of Neuber's rule and the equivalent strain energy density (ESED) method.," International Journal of Fatigue, vol. 26, pp. 447-455, 2004. 


\section{Figure Captions}

Figure 1 Graphical illustration of the left hand side of Eqs (2a) and (2b).

Figure 2 Graphical illustration of the left hand side of Eq. (5).

Figure 3 Graphical illustration of the left hand side of Eqs (2b) and (4).

Figure 4 Graphical illustration of the left hand side of Eq. (6).

Figure 5 Graphical illustration of the left hand side of Eq. (7).

Figure 6 Graphical illustration of two different $\phi$ versus the ratio of $G_{S} / G$.

Figure 7 A graphical illustration of the FE model showing (a) Quadratic Hex (QH) element (b) out-of-plane shear loading [9].

Figure 8 The left graphs $(\mathrm{a}-\mathrm{i})$ depict the normalized nominal shear stress versus notch shear strain. The right graphs $(j-r)$ depict the normalized nominal shear stress versus percentage error with respect to FEA results.

Figure 9 The graphs depict the normalized nominal shear stress versus percentage error with respect to FEA results. 
fig. 1

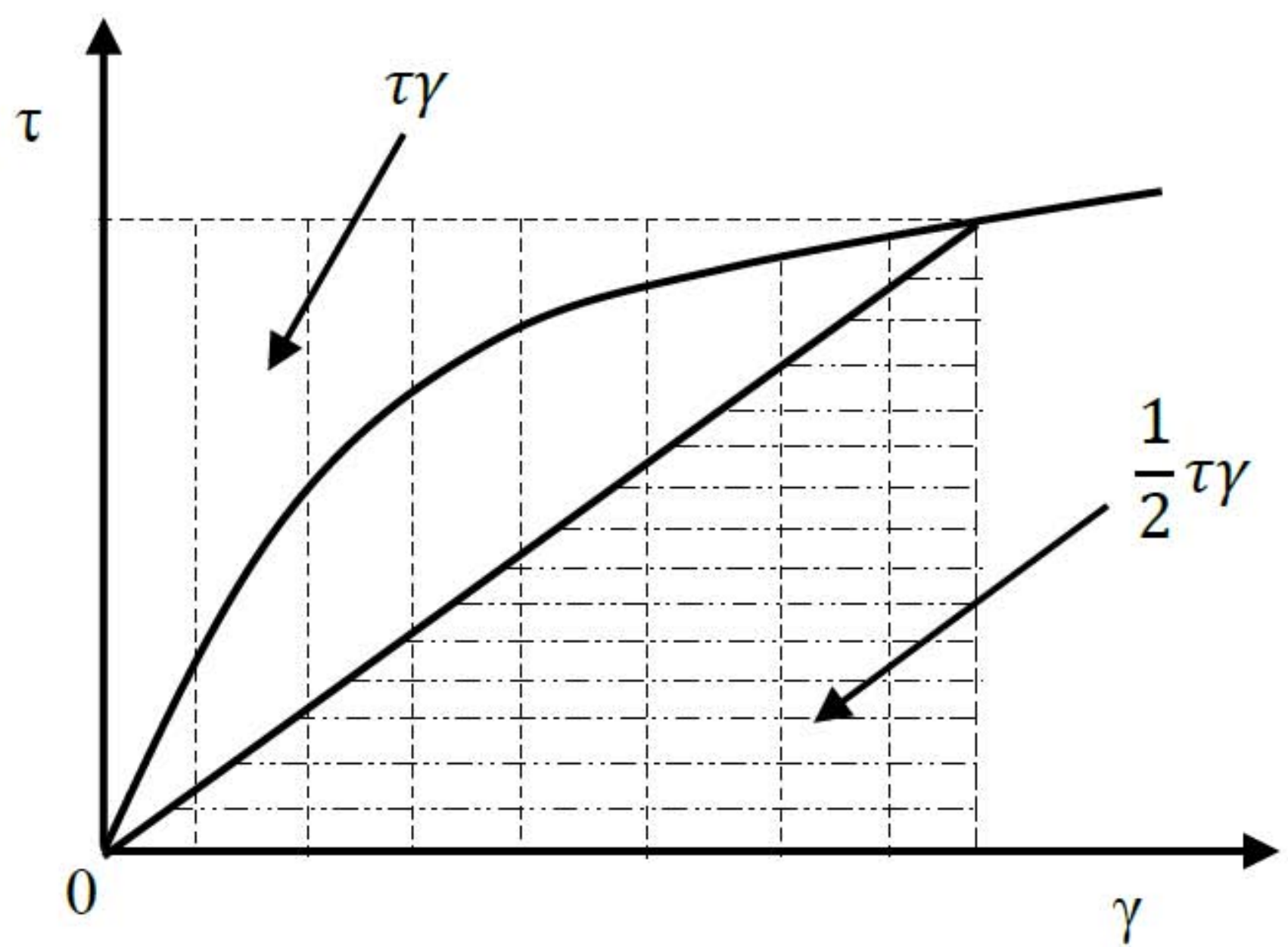


fig. 2

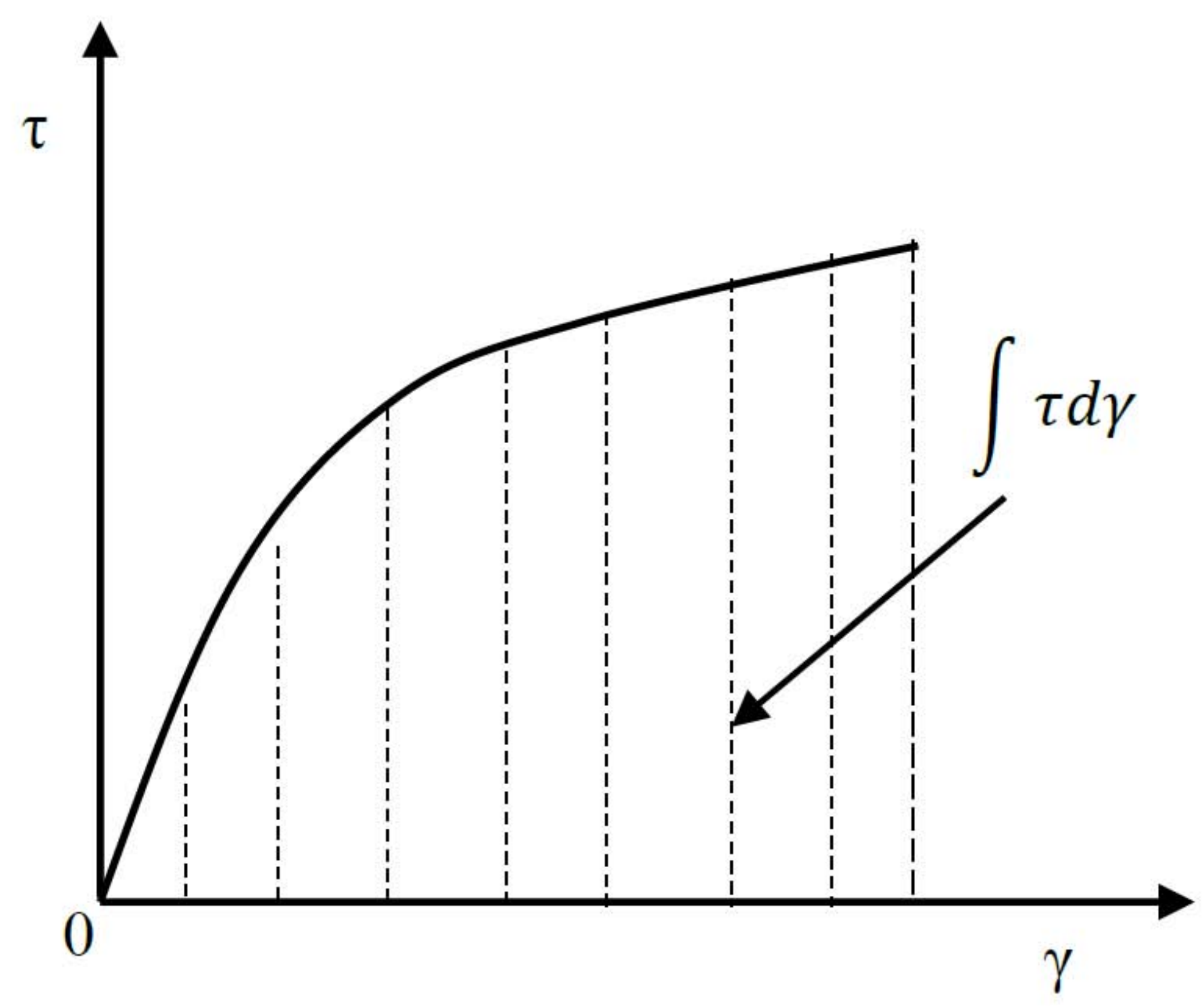


fig. 3

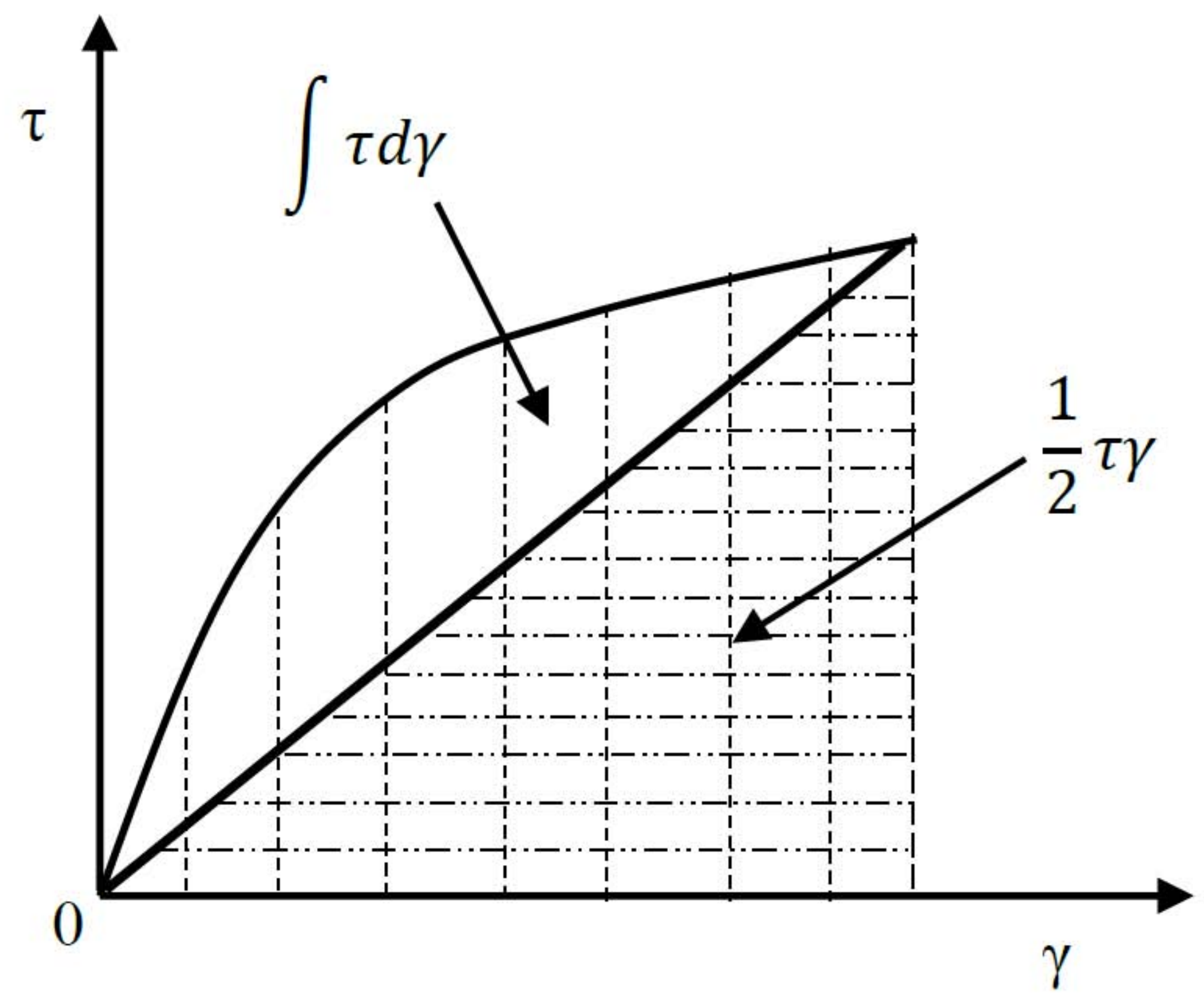


fig .4

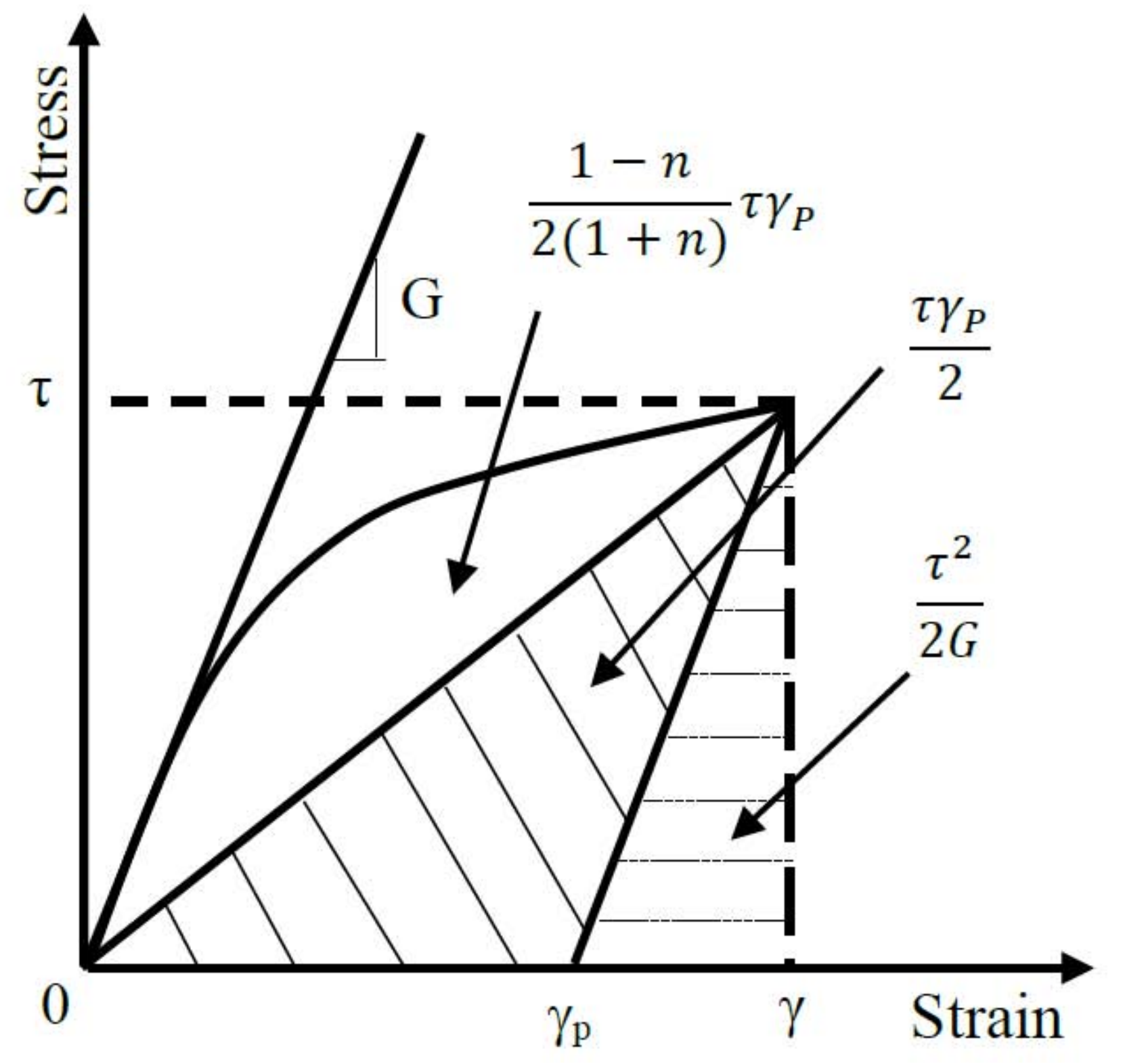


fig. 5

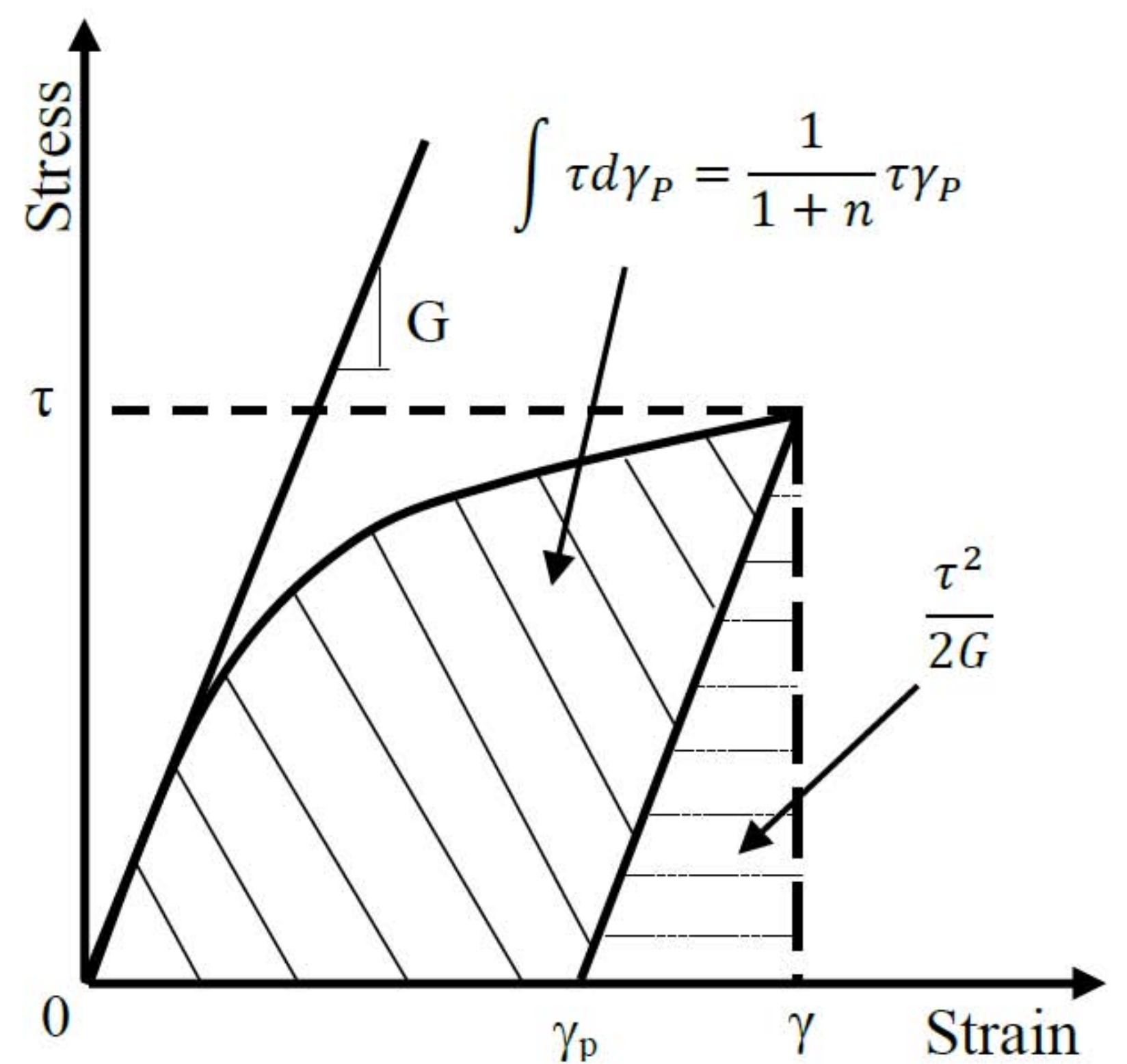




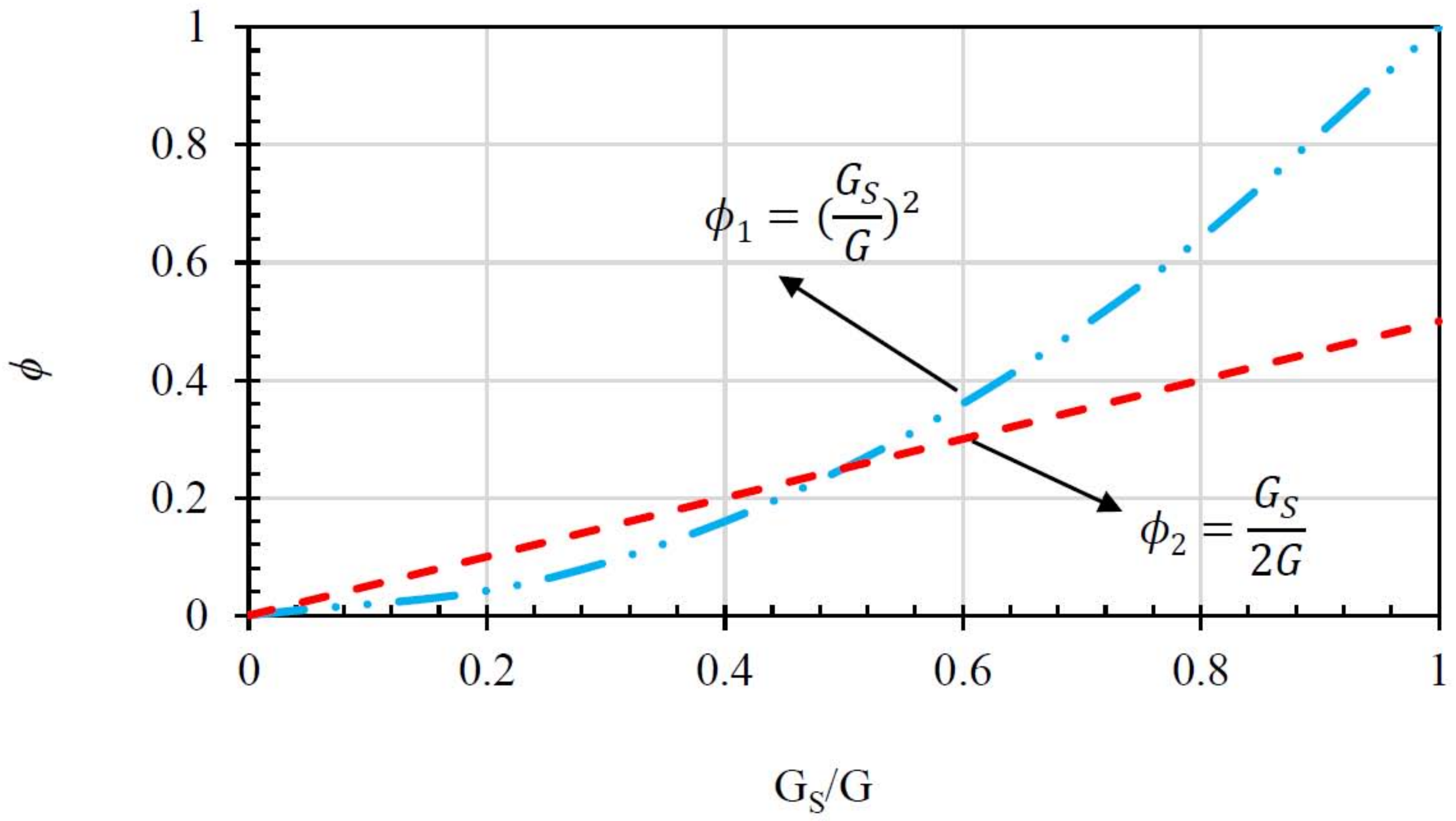


fig .7

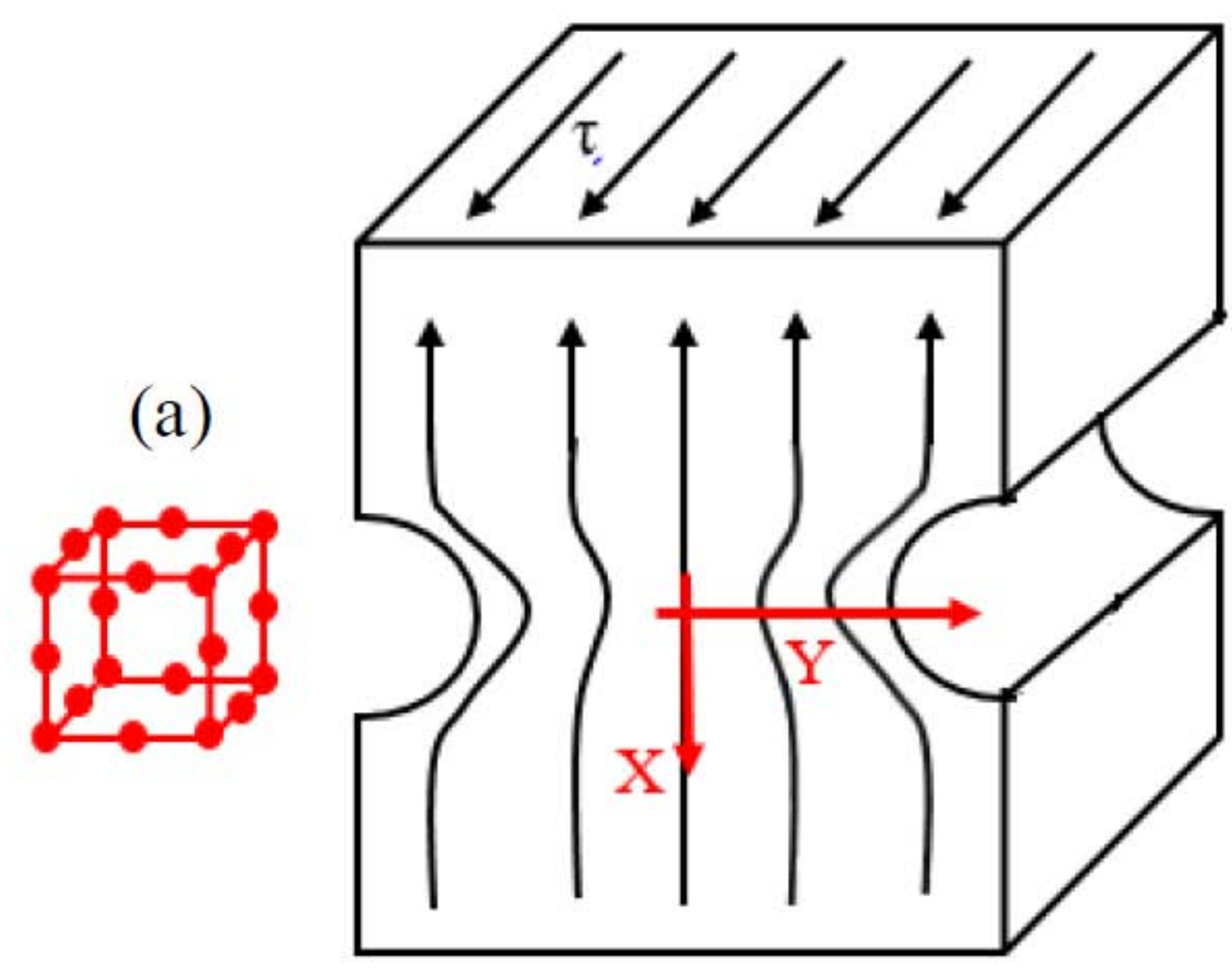

(b) 

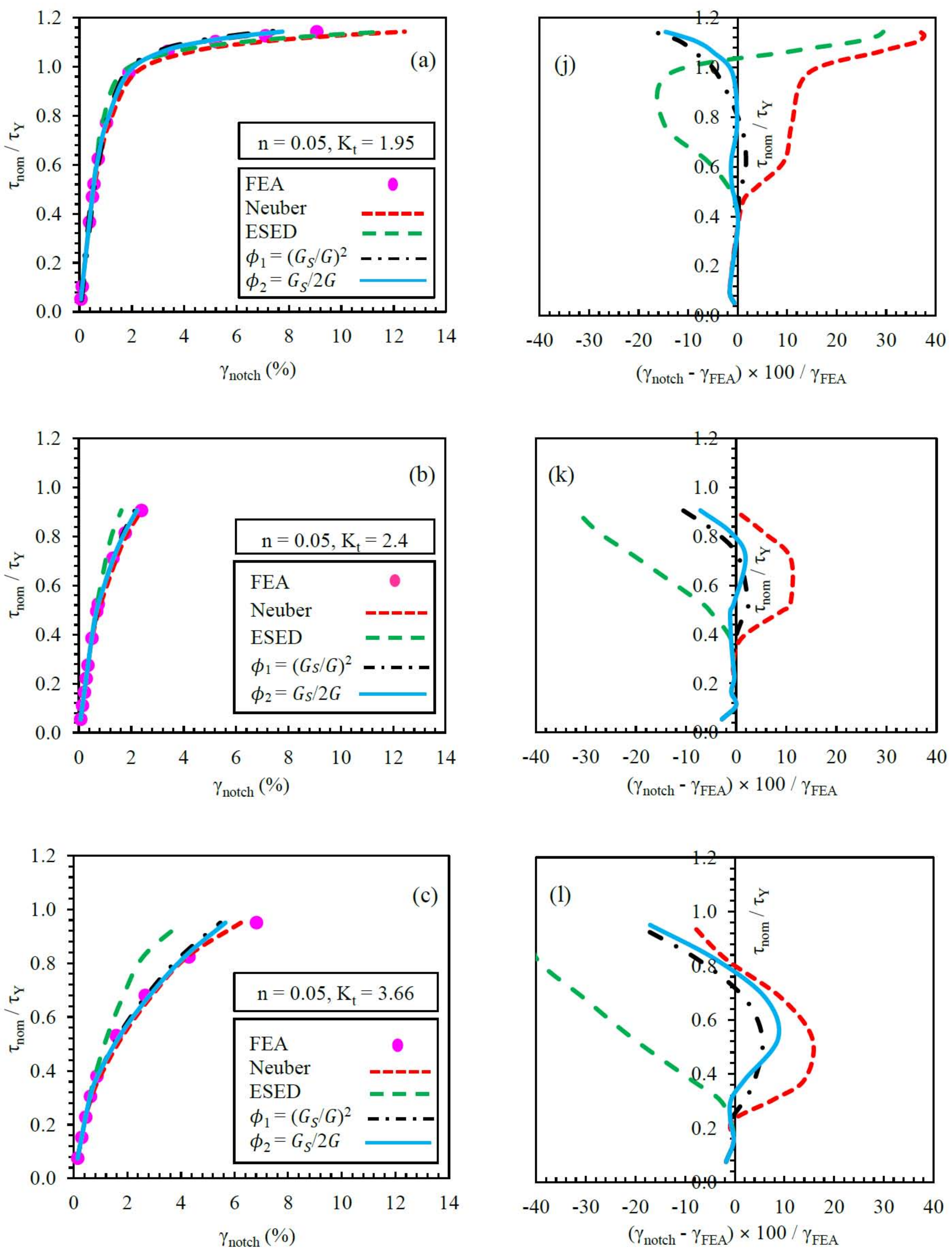

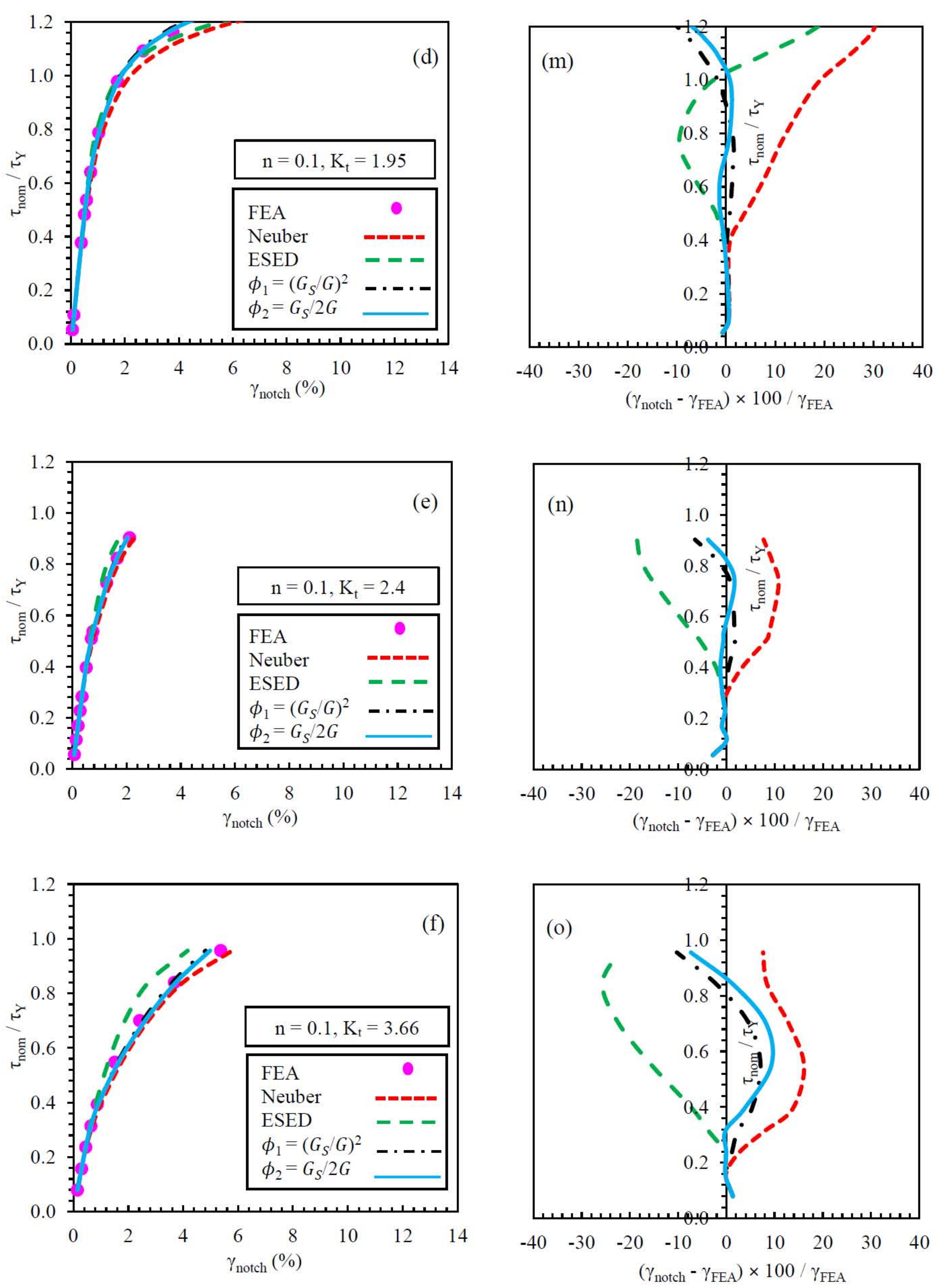

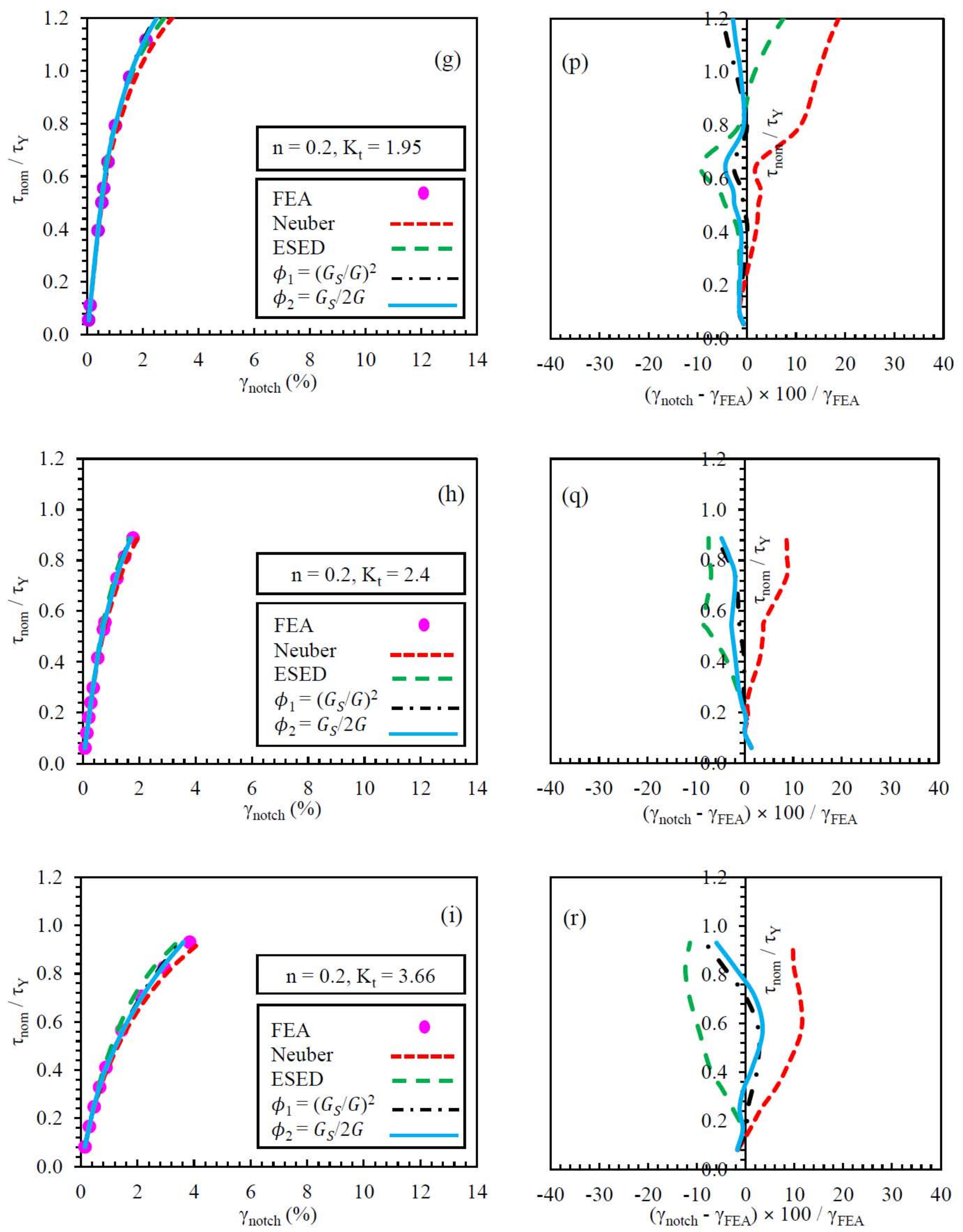
Neuber

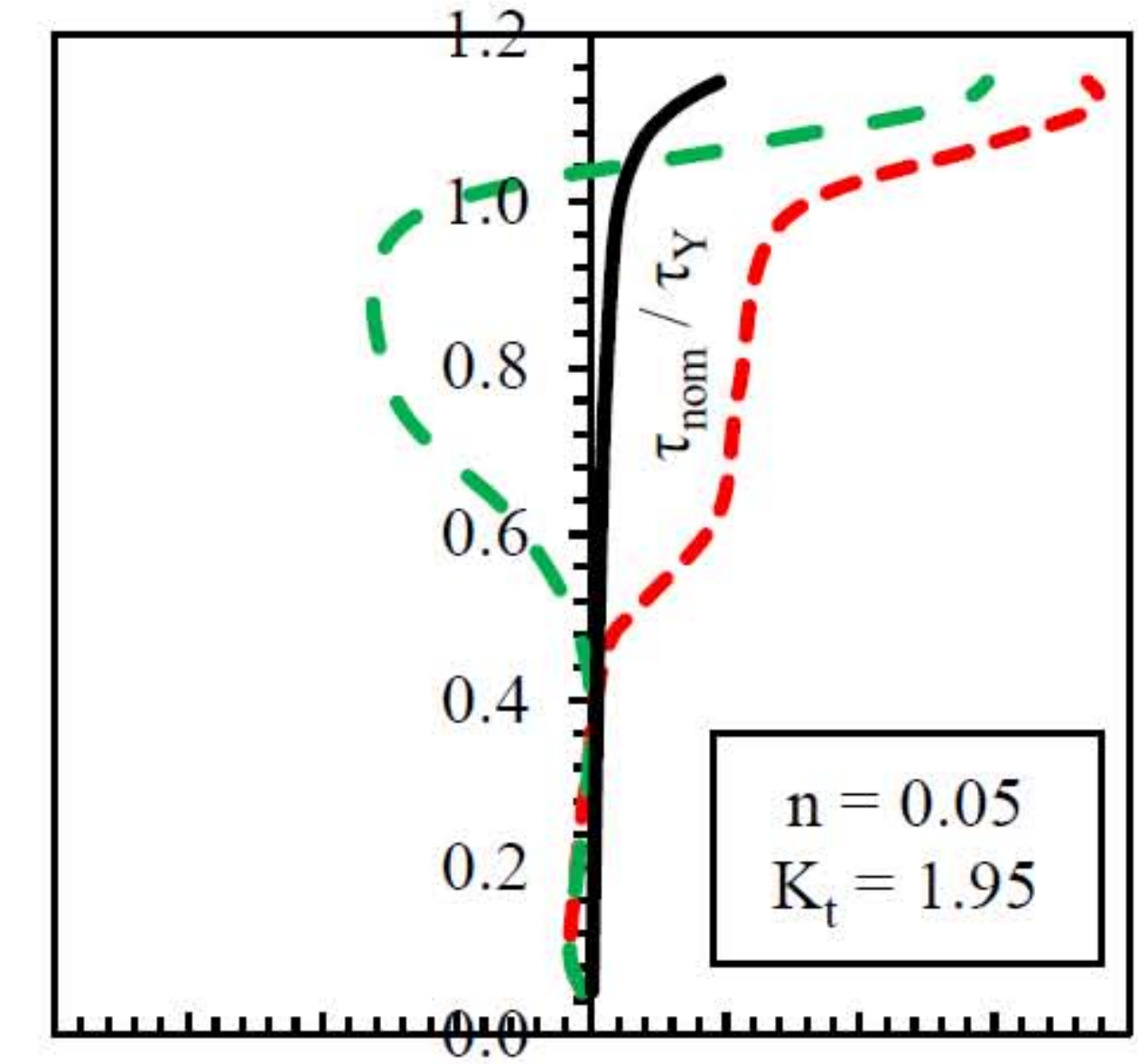

$\begin{array}{lllllllll}-40 & -30 & -20 & -10 & 0 & 10 & 20 & 30 & 40\end{array}$

$\left(\gamma_{\text {notch }}-\gamma_{\mathrm{FEA}}\right) \times 100 / \gamma_{\mathrm{FEA}}$

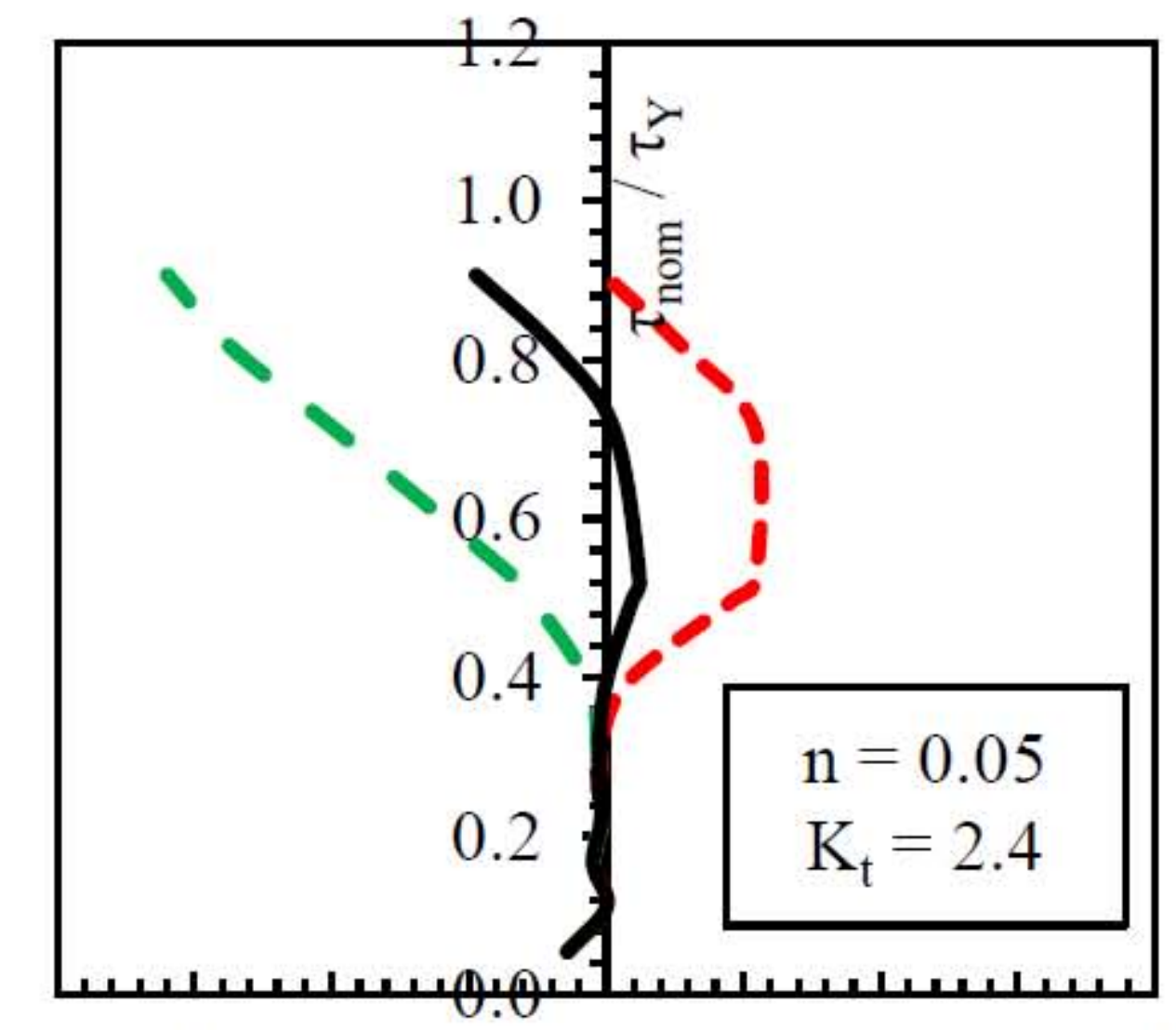

$\begin{array}{lllllllll}-40 & -30 & -20 & -10 & 0 & 10 & 20 & 30 & 40\end{array}$

$\left(\gamma_{\text {notch }}-\gamma_{\mathrm{FEA}}\right) \times 100 / \gamma_{\mathrm{FEA}}$

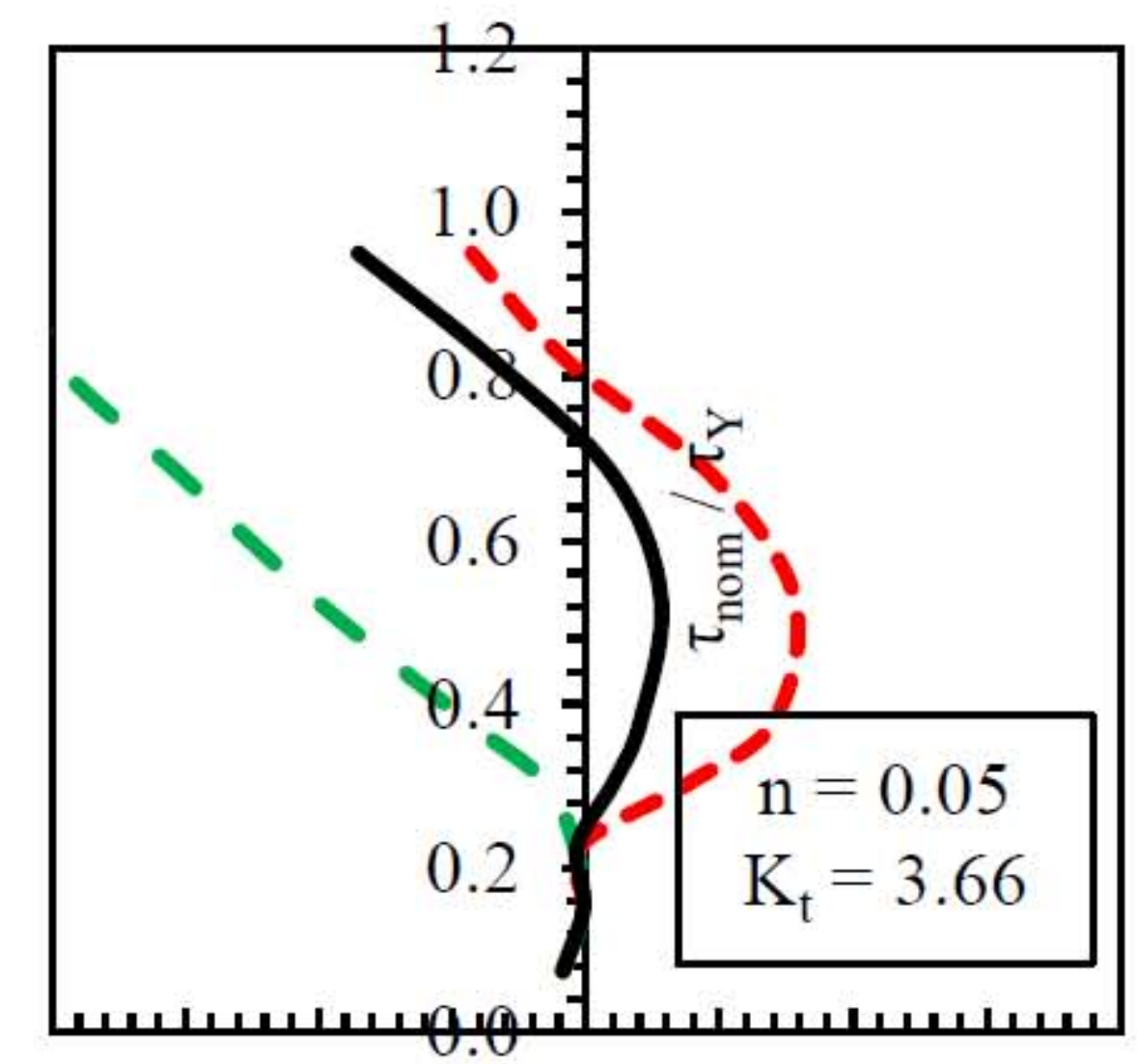

$\begin{array}{lllllllll}-40 & -30 & -20 & -10 & 0 & 10 & 20 & 30 & 40\end{array}$

$\left(\gamma_{\text {notch }}-\gamma_{\mathrm{FEA}}\right) \times 100 / \gamma_{\mathrm{FEA}}$
ESED -

Eq. (16)
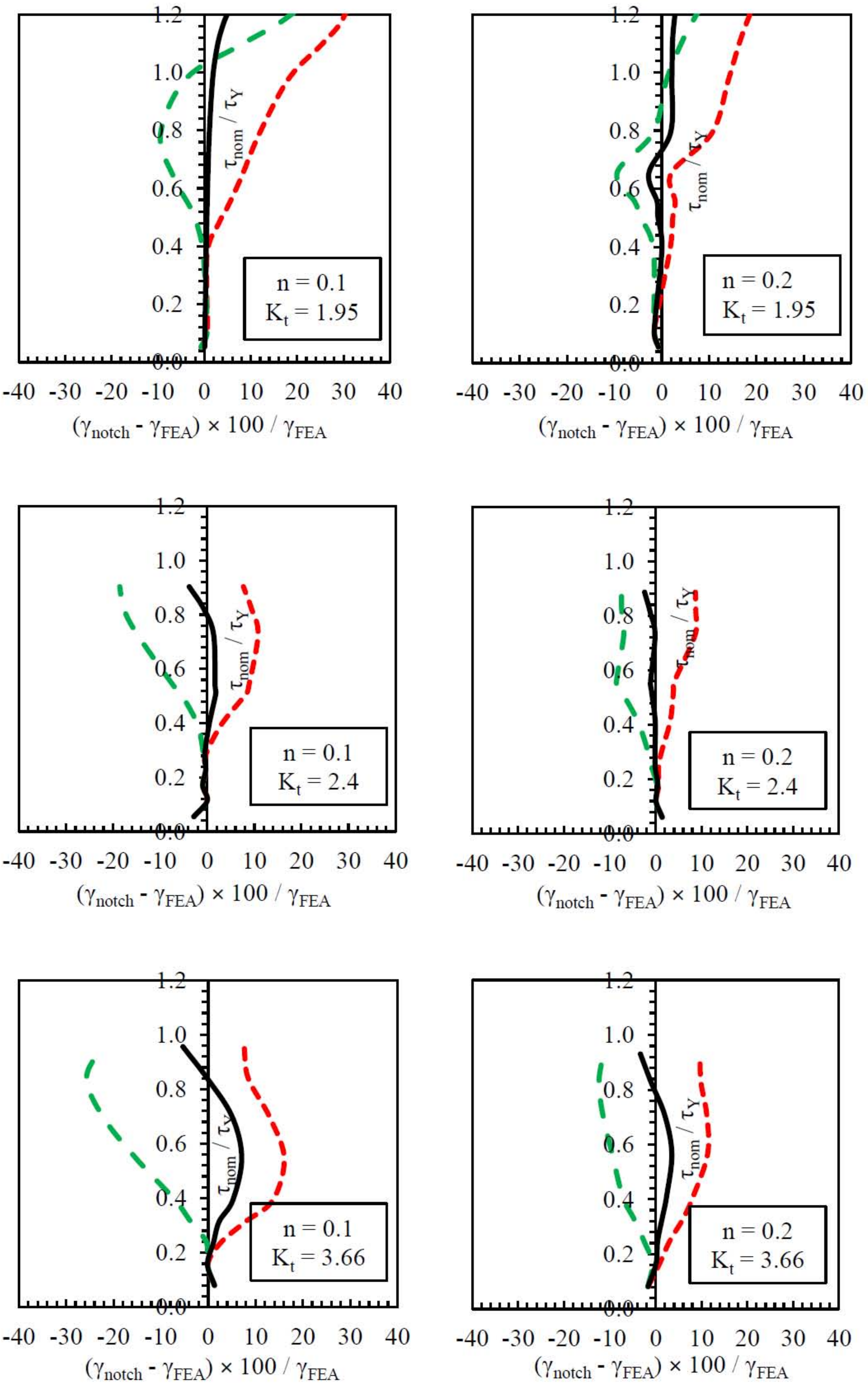